(c) American Dairy Science Association, 2003.

\title{
ADSA Foundation Scholar Award- An Integrated Science-Based Approach to Dairy Food Safety: Listeria monocytogenes as a Model System
}

\author{
M. Wiedmann \\ Department of Food Science, \\ Cornell University, \\ Ithaca, NY 14853
}

\begin{abstract}
Transmission of food- and milkborne pathogens often involves complex interactions among the pathogen, the environment, and one or multiple host species. A complete understanding of these interactions is critical to allow the development of science-based, effective intervention strategies for foodborne infectious diseases. This article summarizes our studies on the transmission, ecology, pathogenesis and population genetics of Listeria monocytogenes, which we have used as model for a food- and milkborne pathogen that infects multiple hosts and also has considerable ability to survive and multiply in nonhost environments. Application of molecular subtyping tools in conjunction with phenotypic characterization of selected strains has allowed us to define distinct $L$. monocytogenes subtypes and clonal groups that appear to differ in relevant phenotypic characteristics that may affect their abilities to be transmitted through food systems. For example, a genetic group designated as lineage I has been shown to be not only more common among human listeriosis cases than among animal cases, but lineage I strains also appear to show an increased in vitro ability to spread intracellularly from host cell to host cell. These findings are consistent with the fact that while genetically diverse strains may be classified to one bacterial species, these strains often differ from one another in important genetic and phenotypic characteristics. I thus propose that evolutionary- and molecular subtyping-based definitions of bacterial subtypes and clonal groups will provide critical insight into the microbial ecology of dairy food systems, including not only foodborne pathogens, but also organisms important for dairy fermentation and spoilage.

(Key words: dairy food safety, Listeria monocytogenes, ecology, population genetics)
\end{abstract}

Received August 12, 2002.

Accepted December 6, 2002.

\begin{abstract}
Abbreviation key: $\mathbf{C D C}=$ Centers for Disease Control and Prevention, MEE = multilocus enzyme electrophoresis.
\end{abstract}

\section{INTRODUCTION}

A recent estimate by researchers at the US Centers for Disease Control and Prevention (CDC) projects that 76 million cases of gastrointestinal foodborne illnesses caused by viral, bacterial, protozoal, and parasitic pathogens occur annually in the United States. These illnesses are estimated to lead to approximately 325,000 hospitalizations and 5000 deaths per year in the United States (Mead et al., 1999). Worldwide, foodborne pathogens also play an important role as a cause of human infectious diseases (WHO, 1999). Thus, foodborne illnesses and their related medical sequelae have a significant negative impact on human health and well being. While estimates are available on the overall incidence of foodborne disease in the United States, estimates on the frequency of foodborne infections linked to consumption of dairy products are not easily available, particularly because a large proportion of foodborne diseases and infections is not tracked back to a specific food source. The incidence of milkborne illnesses in the United States has decreased considerably over the last six to seven decades, however. Whereas it is reported that $25 \%$ of food- and waterborne disease outbreaks were milkborne in 1938, the 1999 revision of the Pasteurized Milk Ordinance (Public Health Service/Food and Drug Administration, 1999) states that less than $1 \%$ of total reported foodborne disease outbreaks are currently linked to milk and milk products. Nevertheless, even in the last two decades, large foodborne disease outbreaks have been linked to dairy products, including outbreaks due to Listeria monocytogenes contaminated Hispanic-style cheeses and due to Salmonella contaminated fluid milk (Boor, 1997, 2001; Anonymous, 2001).

Changes in food production and distribution practices may affect the microbial ecology in food processing systems and the transmission of foodborne diseases. Simi- 
larly, changes in food consumption patterns, as well as in the human population (such as an increase in the number of elderly and immunocompromised people) can alter patterns of foodborne infections. Separately, or together, changing factors that affect our food system may furthermore contribute to the emergence or reemergence of newly recognized foodborne pathogens (Tauxe, 1997). Improved diagnostic methods also may lead to the discovery of pathogens that had been previously unrecognized as causes of foodborne infections. Continuous efforts to better understand and control transmission of foodborne pathogens in the dairy food system are thus key to assure safe dairy foods for the future.

Infections with foodborne pathogens are a consequence of the interactions of a given pathogen, the environment, and the host. While some foodborne pathogens may be predominantly associated with a single host (the human), a considerable number of foodborne pathogens have the ability to cause disease in a variety of hosts, often in farm animals as well as in humans. Highly host-adapted foodborne and waterborne pathogens (e.g., Vibrio cholerae, Salmonella Typhi) are generally more easily controlled than pathogens that have the ability to infect multiple hosts and/or have the ability to effectively survive and multiply outside a human host. Our capabilities for controlling multihost foodand milkborne pathogens are dependent on the thoroughness of our understanding of their ecology and pathogenesis in different hosts and nonhost environments. Only this basic knowledge will allow the development of science-based, effective, and economical food safety measures from farm to table. As reviewed below, my laboratory has chosen the foodborne pathogen $L$. monocytogenes as a model system to probe the pathogenesis and transmission of foodborne pathogens. We hope that our studies, together with ongoing work in many other laboratories, will ultimately provide a framework for the development and implementation of science-based food safety assurance strategies, not only for $L$. monocytogenes, but also for other pathogens.

\section{LISTERIA MONOCYTOGENES AND LISTERIOSIS}

Listeria monocytogenes is a facultative intracellular gram-positive bacterium associated with potentially serious invasive diseases in humans and in more than 40 animal species (Seeliger, 1961). Infection in these hosts can result in abortion, septicemia, meningitis, and encephalitis as well as other less severe manifestations, including diarrhea and skin infections. Among humans, immunocompromised persons, pregnant women, the elderly, and neonates are particularly at risk of listerial infections. The frequency of clinical human listeriosis in most developed countries is estimated to range between 2 to 15 cases/million population with case mortality rates between 13 and 34\% (Farber and Peterkin, 1991). The vast majority (99\%) of human Listeria infections is thought to be foodborne (Mead et al., 1999). Thus, although foodborne listeriosis cases are less common than many other foodborne diseases (e.g., those caused by E. coli O157:H7, Campylobacter jejuni, or Salmonella), they represent the second most common known cause of fatal human foodborne infections, next only to Salmonella infections.

\section{The Genus Listeria}

In addition to $L$. monocytogenes the genus Listeria also includes Listeria ivanovii, Listeria seeligeri, Listeria innocua and Listeria welshimeri. All members of this genus are gram-positive nonspore-forming rods, closely related to the genera Lactobacillus and Streptococcus. The G $+\mathrm{C} \%$ DNA content of $L$. monocytogenesis about $40 \%$. Whereas L. monocytogenes causes both human and animal disease, $L$. ivanovii is predominantly associated with disease (specifically abortions) in sheep, and human clinical infections with this organism appear to be extremely rare (Cummins et al., 1994; Wesley, 1999). The other Listeria species are considered nonpathogenic. The pathogenic species $L$. monocytogenes and $L$. ivanovii are both hemolytic, as is the nonpathogenic $L$. seeligeri, while $L$. innocua and $L$. welshimeri are nonhemolytic. Listeria monocytogenes can be separated into 13 different serotypes (1/2a, 1/2b, 1/ 2c, 3a, 3b, 3c, 4a, 4ab, 4b, 4c, 4d, 4e, and 7), all of which appear to have been associated with animal disease, although some of them are less commonly isolated from affected animals than others (Wiedmann and Evans, 2002). Generally serotypes $1 / 2 \mathrm{a}, 1 / 2 \mathrm{~b}$, and $4 \mathrm{~b}$ appear to be most common among animal isolates, although serotype $4 \mathrm{c}$ is also occasionally found. There are also some indications that the frequency of different serotypes and molecular subtypes among human and animal clinical cases differs. Thus, certain subtypes may show at least some level of host specificity for humans and animals (Wiedmann et al., 1997).

\section{Physiology and Ecology of Listeria}

All Listeria spp. are catalase positive, oxidase negative, and esculin hydrolysis positive. The members of the genus Listeria are aerobic, but also grow under anaerobic conditions and can thus be classified as facultative anaerobes. Listeria monocytogenes has the ability to grow and survive under a variety of different conditions. For example, L. monocytogenes grows from close to 0 to $44^{\circ} \mathrm{C}$ and is thus considered psychrotolerant. 
All Listeria spp. are heat sensitive and pasteurization effectively kills listeria. When cultured at 20 to $25^{\circ} \mathrm{C}$, Listeria is motile due to peritrichous flagella. In media, Listeria grows in a $\mathrm{pH}$ range from 4.4 to 9.6, with optimal growth at neutral $\mathrm{pH}$. L. monocytogenes has a high level of osmotolerance, it can grow in media containing up to $10 \%$ (wt/vol) $\mathrm{NaCl}$, and it can survive at even higher salt concentrations. Perhaps due to its considerable resistance to a variety of stress conditions, L. monocytogenes appears to be able to multiply and/or survive for extended time periods (up to more than $2 \mathrm{yr}$ ) outside mammalian hosts. Consequently it has been isolated from a variety of different environments (e.g., soil, surface water, sewage, vegetative materials, different food processing environments) (Gravani, 1999), and many authors thus consider this organism "ubiquitous."

Because L. monocytogenes represents a foodborne pathogen, a considerable numbers of studies and surveys on the presence of this organism in many raw and ready-to-eat foods have been published. Whereas the prevalence of $L$. monocytogenes varies tremendously by study, type of food surveyed, and other factors, this organism has been isolated from many different foods, occasionally at fairly high prevalences ( $>5$ to $10 \%$ ) (Farber and Peterkin, 1991; Farber and Peterkin, 1999). A summary of raw milk surveys for the presence of $L$. monocytogenes conducted in many different countries indicates that overall $<5 \%$ of raw milk samples are positive for L. monocytogenes (Ryser, 1999b). Most recent surveys of ready to-eat foods in developed countries show $L$. monocytogenes prevalences considerably below 5\%, however, including for dairy foods (Ryser, $1999 \mathrm{a}, 1999 \mathrm{~b})$. The ability of $L$. monocytogenes to survive and proliferate well in foods stored at refrigeration temperatures makes this organism a particular concern for the dairy food industry, since low initial contamination levels (possibly even at $<1 \mathrm{cfu} / 25 \mathrm{~g}$ ) may increase to numbers that could present a human health hazard if products are subject to extended refrigerated storage. Consequently, humans appear to be commonly exposed to L. monocytogenes by food ingestion. Based on the 2001 FDA/USDA Draft Listeria monocytogenes risk assessment (Food and Drug Administration and US Department of Agriculture, 2001), the average US consumer is likely to occasionally (possibly once a year) consume as many as $10^{6}$ to $10^{9} \mathrm{cfu}$ of $L$. monocytogenes in a single serving. In spite of this apparent occasional high exposure, only 2500 human listeriosis cases occur annually in the United States. These data indicate that even exposure to high levels of this organism may not result in human disease, even when one considers that the at-risk population for human listeriosis (immunocompromsied people, pregnant women, etc.) represents only a fraction of the total US population.

\section{Cellular and Molecular Pathogenesis}

The cellular processes of $L$. monocytogenes infection have been well characterized, and genetic systems for gene replacement and for monitoring gene expression have been developed. In tissue culture models, the following stages of infection have been identified: 1) internalization of $L$. monocytogenes within the host cell; 2 ) bacterial escape from the host vacuole; 3 ) multiplication of the parasite within the host cell cytoplasm and its movement through the host cytoplasm by virtue of bacterially directed nucleation of host actin filaments; 4) bacterial movement to the host cell surface and extrusion of bacterial cells in pseudopod-like structures; 5) phagocytosis of these pseudopod-like structures by neighboring cells, followed by escape of the bacterium from the resulting double-membrane vacuole, thus allowing the cycle to repeat (Portnoy et al., 1992).

Gene products essential for each step of the infection process have been identified. Six $L$. monocytogenes virulence genes ( $p r f A, p l c A, h l y A, m p l$, act $A$, and $p l c B$ ) are located together in one virulence gene cluster. This 8.8kbp gene cluster is located at a defined position in all L. monocytogenes strains characterized to date, namely between the housekeeping gene $l d h$ and prs. Additional L. monocytogenes virulence-associated genes (e.g., iap, inlA, inlB, inlD, inlE, inlF) are not physically linked to this virulence island (Dramsi et al., 1997; Portnoy et al., 1992). The specific functions for these genes in the Listeria infection process have been well defined. For example, internalin A (InlA), encoded by inlA, interacts with E-cadherin to allow $L$. monocytogenes to induce its own uptake by specific host cells (e.g., epithelial cells) thus facilitating step 1) of the infection process described above (Gaillard et al., 1991; Mengaud et al., 1996a; Mengaud et al., 1996b). ActA, the actA gene product, is a surface protein required for intracellular movement and cell-to-cell spread through bacterially induced actin polymerization (steps 3 and 4 of the infection process) (Brundage et al., 1993).

Listeria ivanovii and $L$. seeligeri contain virulence genes that are highly similar to those found in L. monocytogenes. As in L. monocytogenes, the virulence gene cluster in these species is also located between the two housekeeping genes $l d h$ and prs. Whereas the organization of the $L$. ivanovii virulence gene cluster is very similar to that in $L$. monocytogenes, the $L$. seeligeri virulence gene cluster contains additional and differently organized genes (Kreft et al., 1999). Despite the presence of $L$. monocytogenes virulence gene homologues in L. seeligeri, this species is considered nonpathogenic and has been shown to be avirulent in the murine animal model. In tissue culture studies, $L$. seeligeri does not trigger its own uptake in nonphagocytic 
mammalian host cells and it is unable to escape from the vacuole when internalized by phagocytic cells (Kreft et al., 1999). Listeria innocua and L. welshimeri completely lack the virulence gene homologues found in $L$. monocytogenes, L. ivanovii, and L. seeligeri.

\section{PATHOGENESIS AND ECOLOGY OF FOODBORNE AND ZOONOTIC INFECTIOUS DISEASES}

The pathogenesis of foodborne infectious diseases can involve complex interactions between a bacterial pathogen, a variety of environments and one or multiple host species. The ability of bacterial cells to survive and compete in a variety of environments plays a key role in the pathogenesis and transmission of many foodborne diseases. In addition, selective pressures not associated with mammalian hosts may contribute significantly to the emergence and evolution of virulence characteristics related to the ability of bacteria to effectively infect mammalian hosts. Foodborne pathogens infecting both humans and animals (such as L. monocytogenes) provide ideal model systems for studying the ecology of infectious diseases, including adaptation of clonal groups to specific hosts and nonhost environments as well as virulence gene expression and maintenance of virulence characteristics under widely varying conditions, including those not directly associated with a host.

In a simplified model, transmission patterns of $L$. monocytogenes as well as of other foodborne pathogens may encompass all or some of the following steps and environments: 1) bacterial survival in the environment and in animal feeds (e.g., L. monocytogenes and pathogenic $E$. coli are common environmental contaminants); 2) bacterial survival inside nonvertebrate hosts (e.g., protozoans); 3) establishment of clinical or subclinical infections or carrier states in food animals; 4) shedding of the organism into animal products used for human consumption or secondary contamination of animal products; 5) bacterial survival and/or multiplication in non-host environments under food processing and distribution conditions; and 6) infection of human hosts, including survival of gastric passage and establishment of enteric or systemic infections (see Figure 1).

An improved understanding of the ability of bacterial pathogens, and specifically of different clonal groups of a given pathogen, to adapt to different environments and hosts and to maintain and evolve virulence characteristics in different host- and nonhost-related environments will contribute broadly to our understanding of the ecology and transmission dynamics of bacterial pathogens. For example, it is well established that different Salmonella serotypes (e.g., Salmonella Typhi and Salmonella Typhimurium) differ in the type and
Hypothesized Listeria

Transmission Pathway

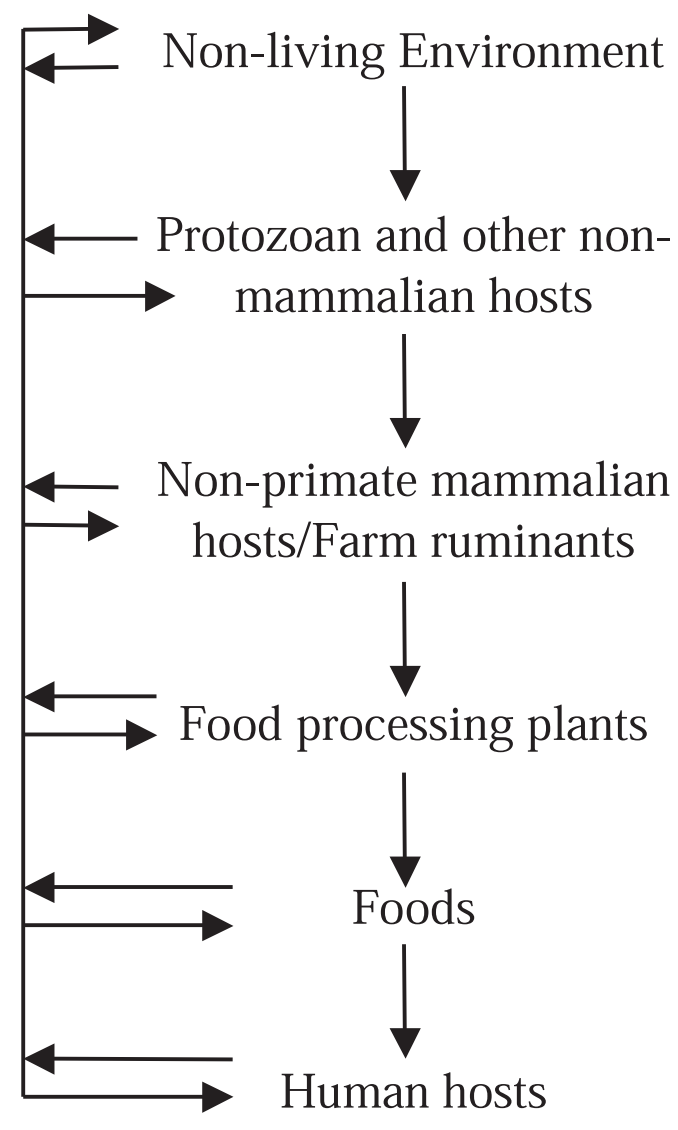

Figure 1. Transmission pathway model for Listeria monocytogenes.

severity of disease they cause in humans, even though they belong to the same bacterial species (Salmonella enterica) (Kingsley and Bumler, 2000). Similarly, it has been recognized that distinct subgroups of the species E. coli differ in their pathogenic potential. For example, Shiga-toxin producing $E$. coli (STEC), which represent a subgroup of all $E$. coli, cause a unique and severe foodborne infection (Acheson, 2000). Although L. monocytogenes can be isolated from a diversity of environmental sources and human and animal hosts, I thus hypothesize that clonal groups of this organism may have adapted to different hosts and nonhost environments.

\section{POPULATIONS GENETICS, TRANSMISSION, AND ECOLOGY OF LISTERIA MONOCYTOGENES}

Current regulations specifying a zero-tolerance for the presence of any L. monocytogenes subtypes in ready- 
to-eat foods are based on historical taxonomic classification schemes. These classical taxonomic definitions of bacterial species do not necessarily correlate with the ability of a group of bacteria to cause human disease. Rather, as outlined above, related bacteria that differ in their abilities to cause human and/or animal disease may be grouped together into the same species. Thus, a critical need exists for the development of better scientific definitions of bacterial groups that have the ability to cause human disease. Molecular subtyping methods provide a unique opportunity to explore the population genetics and evolution of L. monocytogenes (Wiedmann, 2002a). Subtyping methods not only have the potential to differentiate bacterial strains, but also facilitate the definition of subtypes and clonal groups that differ in their phenotypic characteristics and/or their abilities to cause human foodborne disease (Wiedmann, 2002b).

Whereas the majority of human clinical infections occur as sporadic cases, human listeriosis can also occur in large epidemics. Most sporadic human listeriosis cases and large human foodborne listeriosis epidemics have reportedly been caused by $L$. monocytogenes serotype 4b (Farber and Peterkin, 1991; Wiedmann, 2002a). The $4 \mathrm{~b}$ strains isolated from most epidemic outbreaks form two closely related homogenous groups (so called "epidemic clones") (Piffaretti et al., 1989; Wiedmann et al., 1997). Serotypes 1/2a and 1/2b are also responsible for significant numbers of sporadic cases of human illness, and a serotype 1/2a strain was responsible for a recent multistate human listeriosis outbreak in the US (Anonymous, 2000). Serotyping data collected by the CDC in 1986 showed that serotypes 1/2a (30\%), 1/2b $(32 \%)$, and $4 \mathrm{~b}(34 \%)$ represented the majority of isolates from 144 human sporadic cases (Schwartz et al., 1989). Of 1363 human isolates collected in the UK, $15 \%$ were $1 / 2 \mathrm{a}, 10 \%$ were $1 / 2 \mathrm{~b}$, and $64 \%$ were $4 \mathrm{~b}$ (McLauchlin, 1990). The remaining 10 currently recognized L. monocytogenes serotypes have been only rarely linked to human disease. This apparent association between a few specific $L$. monocytogenes strains and most cases of human listeriosis raises the intriguing challenge of identifying unique characteristics enabling these strains to be more effective than others in causing human disease. My research group has thus applied molecular subtyping methods to characterize $L$. monocytogenes isolates from different sources with the goal of defining L. monocytogenes subtypes and clonal groups and their associations with different hosts and nonhost environments. Phenotypic and phylogenetic analyses of selected $L$. monocytogenes strains and isolates are used to probe the functional and evolutionary basis of differences in host distribution and ecology of different clonal groups.

\section{Population Genetics and Evolution of Human, Food, and Animal Listeria monocytogenes Isolates}

Initial studies by my research group and collaborators focused on probing the population genetics of $L$. monocytogenes using isolates from human and animals with clinical listeriosis as well as from contaminated food products. For more than $10 \mathrm{yr}$, our research group has developed a variety of collaborations to collect human clinical and animal L. monocytogenes isolates with the goal of establishing a phylogenetic framework for probing relationships among $L$. monocytogenes strains. We have developed a network for the collection of human and animal L. monocytogenes isolates that includes the health departments in different states (including New York State, New York City, Michigan, and Ohio) and the New York State Veterinary Diagnostic Laboratory at Cornell. Our collection already includes more than 700 human and more than 200 animal isolates and we currently obtain approximately $150 \mathrm{hu}$ man and 20 to 30 additional animal L. monocytogenes isolates/year. Since 1997 we have also collected more than 700 isolates from selected food products and from environmental samples. This strain collection provides a unique resource for our research efforts to understand the population genetics and evolution of $L$. monocytogenes.

Molecular subtyping. Most L. monocytogenes isolates in our strain collection have been characterized by the multiple genetic methods including automated ribotyping using rRNA probes and EcoRI digested chromosomal DNA and allelic analysis of the virulence genes $h l y$, actA, and inlA using PCR-RFLP methods (Wiedmann et al., 1997). These genes encode a hemolysin involved in host cell vacuole lysis, ActA, and internalin A, respectively. Ribotyping is a system for generating DNA fingerprints in which the rRNA operons and surrounding regions are analyzed for polymorphic restriction sites by Southern hybridization. Fingerprints from various organisms differ by molecular weight patterns and relative band intensities. Isolates in our collection have specifically been characterized by a highly standardized and fully automated ribotyping system (RiboPrinter, Qualicon Inc., Wilmington, DE) that includes computerized data analysis (Bruce, 1996; Jeffers et al., 2001). Ribotype analysis specifically identified more than 100 L. monocytogenes EcoRI ribotypes that group into 11 ribotype subsets.

Current efforts are focusing on the development of a WWW-based molecular subtype and strain database to allow rapid exchange of strain characteristics and subtype information. A prototype database is already available at www.pathogentracker.net. We are also developing and implementing DNA sequencing-based 
subtyping methods for L. monocytogenes (Cai et al., 2002) to allow more definitive subtype differentiation. Multilocus DNA sequencing data for $L$. monocytogenes isolates will also provide us with the ability to further and more specifically probe the evolution of Listeria spp. and L. monocytogenes.

L. monocytogenes phylogenetic framework. Initial analysis of the molecular subtyping data described above allowed us to separate L. monocytogenes strains into three distinct genetic lineages, designated I, II, and III (Wiedmann et al., 1997). Some or all of these lineages may represent different $L$. monocytogenes subspecies. Each lineage can be separated into three to four ribotype subsets. Ribotype subsets represent groups of similar ribotypes that share a common ribotype fragment (Bruce et al., 1995). Within each ribotype subset, we found between 5 and 20 distinct genetic subtypes as defined by EcoRI ribotypes and virulence gene PCRRFLP data. Specific virulence gene alleles are generally unique to a specific lineage (Wiedmann et al., 1997). Subtype characterization by a variety of additional molecular subtyping methods performed by other groups (including multilocus enzyme electrophoresis, pulsefield gel electrophoresis; amplified fragment length polymorphism, and DNA sequencing of selected $L$. monocytogenes genes) also confirmed lineages I and II as the two primary divisions of $L$. monocytogenes (Piffaretti et al., 1989; Rasmussen et al., 1991; Mazurier et al., 1992; Brosch et al., 1994; Wiedmann, 2002a). Relatively few lineage III strains have been characterized to date as strains classified into this group represent a rare subgroup of $L$. monocytogenes that appears to be primarily associated with animal hosts (Wiedmann et al., 1997). In addition to our data (Wiedmann et al., 1997), Rasmussen et al. (1995) also reported that DNA sequencing data for selected virulence genes provided evidence for the existence of a third $L$. monocytogenes lineage.

Phylogenetic analyses of partial DNA sequences of the act $\mathrm{A}$ virulence gene from 22 isolates also cluster the strains into three main groups that are identical to the three lineages defined by ribotyping and virulence gene PCR-RFLP analyses. Serotype characterization of $L$. monocytogenes strains was also consistent with our division into three lineages. Genetic lineage showed a direct correlation with serovar clusters: lineage I contained serotypes $1 / 2 \mathrm{~b}, 3 \mathrm{~b}$, and $4 \mathrm{~b}$; lineage II contained serotypes $1 / 2 \mathrm{a}, 1 / 2 \mathrm{c}, 3 \mathrm{a}$, and $3 \mathrm{c}$; lineage III contained serotypes $4 \mathrm{a}$ and 4c (Nadon et al., 2001). Ongoing work in my laboratory now uses sequencing of multiple $L$. monocytogenes genes to further probe the phylogeny of this species. I anticipate that results from these experiments will allow us to further refine our understanding of the population structure of $L$. monocy- togenes and particularly of the strains grouped into lineage III.

In summary, through application of molecular tools, we have been able to define distinct $L$. monocytogenes lineages and clonal groups, and also have found strong evidence suggesting a linkage disequilibrium among the genetic and phenotypic characteristics examined (Wiedmann et al., 1997). We did not find any indications for horizontal gene transfer among the genetic loci investigated. Our preliminary data thus establish a phylogenetic framework for further investigation of virulence differences and habitat specialization among $L$. monocytogenes clonal groups.

Subtype characterization of human outbreak strains. In additional studies, we used ribotype analysis to further probe the phylogenetic characterization of human epidemic L. monocytogenes strains (Jeffers et al., 2001). The majority of human listeriosis cases appear to be sporadic in nature and, therefore, are associated with a variety of different strains. Relatively few large epidemic listeriosis outbreaks have been reported. Listeria monocytogenes strains associated with these epidemic outbreaks are of particular interest as they may represent a clonal group or groups with a unique transmission potential. It is known for other bacterial pathogens that specific clonal "epidemic" groups are responsible for the majority of human cases of specific infectious diseases. Confirming previous reports, we found that two L. monocytogenes ribotypes were associated with the majority of human epidemic outbreaks reported before 1998. Specifically, ribotypes DUP-1038 and DUP-1042 were associated with at least six and four human epidemics, respectively (Jeffers et al., 2001). Both of these clonal groups represent serotype $4 \mathrm{~b}$ strains and are grouped into lineage I. Recent listeriosis outbreaks in the United States (since 1998) appear to be caused by different strains. For example, the strain responsible for a multistate listeriosis outbreak in the United States in the fall of 1998 represents another lineage I ribotype (DUP-1044A) (Anonymous, 1999), and the strain associated with a multistate listeriosis outbreak in the United States in 2000 (Anonymous, 2000 ) is grouped into our lineage II.

\section{Association of $L$. monocytogenes Subtypes and Clonal Groups with Different Hosts}

We have used the phylogenetic framework described above to probe for possible host specificities and virulence differences among $L$. monocytogenes clonal groups using human and animal clinical isolates. Specifically, we assessed whether different clonal groups as defined by lineage, ribotype subset, and ribotype differed in their likelihood to cause human sporadic or epidemic 
listeriosis cases or animal listeriosis. An initial evaluation of linkages between independent phylogenetic and virulence markers in 195 human and animal L. monocytogenes isolates was published in 2001 (Jeffers et al., 2001). Interestingly, the clinical histories of the $L$. monocytogenes isolates suggested differences in pathogenic potentials among the three lineages when the association between classification to lineage and the isolate source (human or animal) was evaluated. We found a statistically significant predominance of human isolates compared with animal isolates among lineage I strains as well as a significant predominance of animal isolates in comparison to human isolates among lineage II and lineage III strains (Jeffers et al., 2001). Specifically, only one human sporadic isolate and no human epidemic isolates were classified to lineage III, while $11 \%$ of animal isolates were assigned to this lineage. This suggests that strains in this lineage may have reduced virulence for humans and/or that they have limited transmission potential from animal to human hosts. Lineage I contained the majority of human outbreak and sporadic isolates, but only $42 \%$ of the animal isolates. These preliminary findings led us to hypothesize that lineage I strains or a subset of lineage I strains have a greater pathogenic potential for humans as compared to strains in the other two lineages. This hypothesis is also supported by the findings of Vines et al. (1992) who reported that perinatal listeriosis is caused more frequently by strains equivalent to those in our lineage I than by strains in lineage II. A report by McLauchlin (McLauchlin, 1990) also found that serotypes $1 / 2 \mathrm{~b}$ and 4b (grouped in lineage I) represent the majority (74\%) of human isolates $(\mathrm{n}=1363)$, and thus further supports our findings. In conclusion, we and others have accumulated strong evidence that $L$. monocytogenes clonal groups differ in their association with human and animal hosts.

\section{Phenotypic Characterization of $L$. monocytogenes Strains and Subtypes}

Phenotypic characterization of selected isolates from our collection has been initiated to probe the functional basis of possible virulence and transmission differences among different $L$. monocytogenes clonal groups and subtypes. Initial tissue culture virulence characterization using a plaque assay in mouse $\mathrm{L}$ cells indicate that lineage II strains may show reduced plaquing efficiency and/or plaque size as compared to lineage I and III strains (Wiedmann et al., 1997), which may be indicative of reduced virulence for lineage II strains. These preliminary tissue culture data are consistent with data from our group and from others (detailed in the proceeding section), which indicate that lineage I strains (which includes serotypes $1 / 2 \mathrm{~b}$ and $4 \mathrm{~b}$ ) appear to be the predominant strains responsible for human listeriosis cases and outbreaks, while lineage II strains (serotypes $1 / 2 \mathrm{a}$ and $1 / 2 \mathrm{c}$ ) appear to be the most common strains isolated from contaminated foods (Norton et al., 2001b). Further experiments are in progress to confirm our current working hypothesis that lineage I (or a subset of strains within this lineage) represents a subgroup with increased human virulence as compared to lineage II, while lineage II shows an increased ability for environmental survival and multiplication as compared to lineage I. Because lineage III strains represent about 10\% of isolates from animals with clinical symptoms, but are considerably less frequent among human clinical isolates and among food isolates (Norton et al., 2001b), these strains may be characterized by a limited transmissibility from animals through foods (and/or environmental; sources) to humans, possibly either due to limited environmental survival and growth capabilities or due to limited human virulence.

\section{Ecology of L. monocytogenes}

Survival of $L$. monocytogenes outside human and animal hosts plays an important role in the transmission of this organism in the dairy food system. While it is conceivable that $L$. monocytogenes can enter the dairy food chain at many and possibly even at nearly any point, agricultural environments and food processing plant environments may be of particular importance as sources for the introduction of $L$. monocytogenes into the dairy food system.

Listeriosis infections in farm animals and particularly in cattle are often linked to consumption of contaminated silage. In addition, animal listeriosis cases sometimes occur in animals that are not fed silage and environmental sources have been speculated to be responsible for at least some of these cases. The agricultural environment thus may serve not only as an important source for contamination of silage, but may also be a direct source of animal infection in some cases.

The role of contaminated raw animal-based agricultural products (e.g., milk, meat) as a direct source of L. monocytogenes contamination of ready-to-eat food products is likely to be minimal since commercially applied heat treatments generally kill $L$. monocytogenes effectively enough to provide an appropriate margin of safety. While, infected animal and agricultural environments appear to rarely be a direct cause of human infections, there have been cases in which a direct link has been observed. Specifically, animal sources can play an important role in animal-derived food products that are not processed before consumption (e.g., raw milk). In addition, manure from infected or shedding animals 
may represent a source of food contamination. For example, an outbreak involving 42 human cases in Nova Scotia in 1981 was linked to the consumption of coleslaw. This coleslaw was produced from cabbage harvested from fields fertilized with untreated sheep manure that had been obtained from a farm with a history of ovine listeriosis (Schlech et al., 1983).

Contaminated raw meat and milk as well as agricultural environments and/or animal fecal materials may also serve as an indirect source of food contamination by contributing to contamination of the food processing plant environment. Increasing evidence indicates that the processing plant environment may be one of the most important sources of food product contamination with $L$. monocytogenes. We thus have focused research efforts on developing a better understanding of the ecology of $L$. monocytogenes in agricultural environments and in food and dairy processing plants to better understand the contributions of these environments to processed food product contamination with $L$. monocytogenes. Further, it is possible that these environments serve as reservoir for this organism. Application of molecular subtyping methods that have been developed over the last 10 to $15 \mathrm{yr}$ provides an opportunity to study of the distribution of $L$. monocytogenes in different environments and to track the sources and spread of specific $L$. monocytogenes subtypes throughout the food chain, allowing insight into the ecology of different L. monocytogenes clonal groups (Wiedmann, 2002a).

Listeria monocytogenes in the agricultural environment. To better understand the ecology of L. monocytogenes, we have initially applied molecular subtyping methods to track the spread and distribution of different $L$. monocytogenes subtypes on farms with listeriosis outbreaks. These studies have found a considerable diversity of $L$. monocytogenes subtypes in feed (primarily silage) samples as well as in other environmental samples collected on outbreak farms. Interestingly, in some outbreaks multiple distinct $L$. monocytogenes subtypes were shown to be responsible for the animal cases (Wiedmann et al., 1996), while in others a single subtype was responsible for all cases observed (Wiedmann et al., 1997). These findings might indicate that listeriosis epizootics can differ with regard to the causative strains and their spread. In some epizootics, a single, possibly highly virulent, strain may be responsible for infection of all involved animals, whereas in others distinctive strains (possibly with lower virulence and/or transmission potential) may be responsible for individual infections (Wiedmann et al., 1997). In many of the listeriosis outbreaks in farm animals investigated by our group, a considerable proportion, and sometimes all, of the $L$. monocytogenes subtypes found on a given farm were only isolated from environmental samples and not from infected animals. These findings could indicate that some environmental isolates may have a limited ability to cause disease and thus further supports that $L$. monocytogenes strains may differ in their virulence characteristics.

Ongoing research in my laboratory focuses on developing a broader understanding of the ecology of $L$. monocytogenes in farm environments (including farms without listeriosis cases) as well as in pristine and urban environments. Initial findings indicate that while L. monocytogenes is indeed found in many environments, including urban and pristine environments, it is considerably more frequently isolated in farm environments (Sauders et al., 2002). Further subtype and phenotypic analyses and virulence characterization of the $L$. monocytogenes isolates from these environments will provide a better understanding of the ecology of this foodborne pathogen outside human and animal hosts.

Listeria monocytogenes in food and dairy processing environments. Because $L$. monocytogenes is effectively inactivated by commercial heat treatments (pasteurization) used in the dairy industry, the food processing environment seems to represent a major source of finished product contamination. Whereas $L$. monocytogenes has been reported to be regularly isolated from food and dairy processing environments (Cox, 1989; Pritchard et al., 1995; Sutherland and Porritt, 1996; Gravani, 1999), a better knowledge of the spread and survival of $L$. monocytogenes in food processing environments and the contributions of different environmental sources to finished product contamination is necessary to understand the transmission of $L$. monocytogenes. Whereas many food processors only test for the presence of Listeria spp. in the processing environment, detection and isolation of $L$. monocytogenes followed by molecular subtype characterization provides an opportunity to study the molecular ecology of L. monocytogenes in food processing plants. A thorough understanding of the molecular ecology of foodborne pathogens is necessary for the design of rational and science-based methods and approaches for the control of $L$. monocytogenes and other foodborne pathogens.

In a pilot study using three smoked fish processing plants, we showed that specific L. monocytogenes subtypes persisted in the environment of a given processing plant (Norton et al., 2001a). In addition to these persistent, plant-specific, subtypes, we also observed a considerable diversity of transient $L$. monocytogenes subtypes. Interestingly, the persistent $L$. monocytogenes subtypes were the major cause of finished product contamination. Through a collaborative research project on the ecology of $L$. monocytogenes in Mexican-style cheese processing plants, we also showed a similar pattern of strain distribution in dairy processing plants, including persistence 
of plant-specific subtypes over time. In one of the three plants studied, the persistent $L$. monocytogenes subtype was also responsible for finished product contamination (Kuaye et al., 2001). These findings are consistent with other reports that used bacterial subtyping methods to show the persistence of specific L. monocytogenes subtypes in a variety of food processing environments including those for smoked fish, poultry, meat and dairy foods ( Lawrence and Gilmour, 1995; Rorvik et al., 1995; Nesbakken et al., 1996; Autio et al., 1999;). Whereas some studies found that persistent strains may have an increased capability to adhere to inorganic surfaces (Lunden et al., 2000), others were not able to discern an association between strain persistence and a strain's ability to form biofilms (Djordjevic et al., 2002). Further research will be necessary to determine whether specific phenotypic and genetic strain characteristics are responsible for persistence in a processing plant environment.

While further confirmation of the association between persistent stains and finished product contamination in other dairy and food processing plants will be important, our results indicate that control and elimination of persistent $L$. monocytogenes subtypes in processing plants may allow for considerable reduction of finished product contamination. Transient $L$. monocytogenes strains on the other hand may only be minor contributors to finished product contamination. Molecular subtyping thus provides an important tool to track in-plant sources and spread of bacterial contaminants and to better control finished product contamination by foodborne pathogens (Wiedmann, 2002a, Wiedmann, 2002b).

\section{CONCLUSIONS}

Transmission of food and milkborne pathogens often involves complex interactions among the pathogen, the environment and one or multiple host species. A complete understanding of these interactions is important to develop science-based and effective intervention strategies to reduce the incidence of foodborne infectious diseases. Within this context, it is important to understand that bacteria grouped into a single species often represent a diverse group of organisms. To illustrate, bacteria sharing as little as 70\% DNA-DNA homology can be grouped into a single species. In our studies using $L$. monocytogenes as a model system, we have found considerable evidence for the existence of distinct $L$. monocytogenes subtypes and clonal groups that appear not only to represent distinct phylogenetic groups, but also to differ in phenotypic characteristics and transmission dynamics. Our findings are consistent with current knowledge of other foodborne pathogens (e.g., Salmonella, E. coli), where strains and clonal groups within a given species can differ considerably in their transmission characteristics and in their ability to cause foodborne disease. I thus propose that rational and science-based approaches to food safety should be based on specific definitions of bacterial subtypes and clonal groups that have the ability to cause human disease, rather than on broad bacterial species definitions, which may not relate to the ability of a group of bacteria to cause human disease. Understanding bacterial diversity beyond the species level thus represents an important task for all food and dairy microbiologists. Strain differences among microbial organisms grouped into a single bacterial species are not only important for our understanding of bacterial pathogens, but these differences also play important roles for spoilage organisms and starter cultures. While it may be difficult to imagine dairy microbiology without the species and taxonomic units we are familiar with, a move towards evolutionary- and molecular subtyping-based definitions of bacterial and clonal groups will improve our understanding of microbes associated with the dairy food system. These ideas can be summarized best with a quote from Norman Pace, a renowned microbiologist: "I think the concept of species is a fallacy in the microbial world" (Stencel, 2000).

\section{ACKNOWLEDGMENTS}

Research in the author's laboratory has been supported by 1) the National Oceanic and Atmospheric Administration award \#NA86RG0056 to the Research Foundation of State University of New York for New York Sea Grant; 2) USDA-NRI under award no.9935201-8074; 3) the National Institutes of Health Award No. R01GM63259 (to M.W.); and 4) the North American Branch of the International Life Sciences Institute (ILSI N.A.). The US government is authorized to produce and distribute reprints for governmental purposes notwithstanding any copyright notation that may appear hereon. Any opinions, findings, conclusions, or recommendations expressed in this publication are those of the author and do not necessarily reflect the views of NOAA, NIH, USDA or any of their subagencies, or ILSI.

\section{REFERENCES}

Acheson, D. 2000. How does Escherichia coli O157:H7 testing in meat compare with what we are seeing clinically? J. Food Prot. 63:819-821.

Anonymous. 1999. Update: Multistate outbreak of listeriosis-United States, 1998-1999. Morb. Mortal. Wkly. Rep. 47:1117-1118.

Anonymous. 2000. Multistate outbreak of listeriosis-United States, 2000. Morb. Mortal. Wkly. Rep. 49:1129-1130.

Anonymous. 2001. Outbreak of listeriosis associated with homemade Mexican-style cheese-North Carolina, October 2000--January 2001. Morb. Mortal. Wkly. Rep. 50:560-562. 
Autio, T., S. Hielm, M. Miettinen, A.-M. Sjoberg, K. Aarnisalo, J. Bjorkroth, T. Mattila-Sandholm and H. Korkeala. 1999. Sources of Listeria monocytogenes contamination in a cold-smoked rainbow trout processing plant detected by pulsed-field gel electrophoresis typing. Appl. Environ. Microbiol. 65:150-155.

Boor, K . 1997. Pathogenic microorganisms of concern to the dairy industry. Dairy Food Environ. Sanit. 17:714-717.

Boor, K. 2001. Fluid dairy product quality and safety: Looking to the future. J. Dairy Sci. 84:1-11.

Brosch, R., J. Chen, and J. B. Luchansky. 1994. Pulsed-field fingerprinting of listeriae: identification of genomic divisions for Listeria monocytogenes and their correlation with serovar. Appl. Environ. Microbiol. 60:2584-2592.

Bruce, J. 1996. Automated system rapidly identifies and characterizes microorganisms in food. Food Technol. 50:77-81.

Bruce, J. L., R. J. Hubner, E. M. Cole, C. I. McDowell, and J. A. Webster. 1995. Sets of EcoRI fragments containing ribosomal RNA sequences are conserved among different strains of Listeria monocytogenes. Proc. Natl. Acad. Sci. USA 92:5229-5233.

Brundage, R. A., G. A. Smith, A. Camilli, J. A. Theriot, and D. A. Portnoy. 1993. Expression and phosphorylation of the Listeria monocytogenes ActA protein in mammalian cells. Proc. Natl. Acad. Sci. USA 90:11890-11894.

Cai, S., D. Y. Kabuki, A. Y. Kuaye, T. Cargioli, M. Chung, and M. Wiedmann. 2002. Rational design of DNA sequencing-based subtyping methods for Listeria monocytogenes. J. Clin. Microbiol. 40:3319-3325.

Cox, L. J., T. Kleiss, J. L. Cordier, C. Cordellana, P. Konkel, C. Pedrazzini, R. Beumer, and A. Siebenga. 1989. Listeria spp. in food processing, non-food and domestic environments. Food Microbiol. 6:49-61.

Cummins, A. J., A. K. Fielding, and J. McLauchlin. 1994. Listeria ivanovii infection in a patient with AIDS. J. Infect. Dis. 28:89-91.

Djordjevic, D., M. Wiedmann, and L. A. McLandsborough. 2002. Microtiter plate assay for assessment of Listeria monocytogenes biofilm formation. Appl. Environ. Microbiol. 68:2950-2958.

Dramsi, S., P. Dehoux, M. Lebrun, P. L. Goossens and P. Cossart. 1997. Identification of four new members of the internalin multigene family of Listeria monocytogenes EGD. Infect. Immun. 65:1615-1625.

Farber, J. M., and P. I. Peterkin. 1991. Listeria monocytogenes, a food-borne pathogen. Microbiol. Rev. 55:476-511.

Farber, J. M., and P. I. Peterkin. 1999. Incidence and behaviour of Listeria monocytogenes in meat products. Pages 505-564 in Listeria, Listeriosis, and Food Safety. E. T. Ryser and E. H. Marth, eds. Marcel Dekker, Inc., New York.

Food and Drug Administration and US Department of Agriculture. 2001. Draft assessment of the relative risk to public health from foodborne Listeria monocytogenes among selected categories of Ready-to-Eat foods. USDA, FDA, Washington DC (http:// www.foodsafety.gov/ dms/lmrisk.html).

Gaillard, J.-L., P. Berche, C. Frehel, E. Gouin and P. Cossart. 1991. Entry of L. monocytogenes into cells is mediated by internalin, a repeat protein reminiscent of surface antigens from gram-positive cocci. Cell 65:1127-1141

Gravani, R. 1999. Incidence and control of Listeria in food-processing facilities. Pages 657-709 in Listeria, Listeriosis, and Food Safety. E. T. Ryser and E. H. Marth, ed. Marcel Dekker, Inc., New York.

Jeffers, G. T., J. L. Bruce, P. McDonough, J. Scarlett, K. J. Boor, and M. Wiedmann. 2001. Comparative genetic characterization of Listeria monocytogenes isolates from human and animal listeriosis cases. Microbiology 147:1095-1104.

Kingsley, R. A., and A. J. Bäumler. 2000. Host adaptation and the emergence of infectious disease: The Salmonella paradigm. Mol. Microbiol. 36:1006-1014.

Kreft, J., J.-A. Vazquez-Boland, E. Ng, and W. Goebel. 1999. Virulence gene clusters and putative pathogenicity islands in Listeriae. Pages 219-232 in Pathogenicity Islands and Other Mobile Virulence Elements. J. B. Kaper and J. Hacker, eds. American Society for Microbiology Press, Washington, DC.
Kuaye, A. Y., D. Y. Kabuki, M. Wiedmann, and K. J. Boor. 2001. Molecular subtyping and tracking of Listeria monocytogenes in Hispanic cheese factories. J. Dairy Sci. 84(Suppl. 1):256. (Abstr.) Lawrence, L. M., and A. Gilmour. 1995. Characterization of Listeria monocytogenes isolated from poultry products and from the poultry-processing environment by random amplification of polymorphic DNA and multilocus enzyme electrophoresis. Appl. Environ. Microbiol. 61:2139-2144.

Lunden, J., M. Miettinen, T. Autio, and H. Korkeala. 2000. Persistent Listeria monocytogenes strains show enhanced adherence to food contact surface after short contact times. J. Food Prot. 63:1204-1207.

Mazurier, S.-I., A. Audurier, N. M.-V. d. Mee, S. Notermans, and K. Wernars. 1992. A comparative study of randomly amplified polymorphic DNA analysis and conventional phage typing for epidemiological studies of Listeria monocytogenes isolates. Res. Microbiol. 143:507-512.

McLauchlin, J. 1990. Distribution of serovars of Listeria monocytogenes isolated from different categories of patients with listeriosis. Eur. J. Clin. Microbiol. Infect. Dis. 9:210-213.

Mead, P., L. Slutsker, V. Dietz, L. F. McCaig, J. S. Bresee, C. Shapiro, P. Griffin, and R. V. Tauxe. 1999. Food-related illness and death in the United States. Emerg. Infect. Dis. 5:607-625.

Mengaud, J., M. Lecuit, M. Lebrun, F. Nato, J.-C. Mazie, and P. Cossart. 1996a. Antibodies to the leucine-rich repeat region of internalin block entry of Listeria monocytogenes into cells expressing E-cadherin. Infect. Immun. 64:5430-5433.

Mengaud, J., H. Ohayon, P. Gounon, R.-M. Mege, and P. Cossart. 1996b. E-Cadherin is the receptor for internalin, a surface protein required for entry of $L$. monocytogenes into epithelial cells. Cell 84:923-932.

Nadon, C. A., D. L. Woodward, C. Young, F. G. Rodgers, and M. Wiedmann. 2001. Correlations between molecular subtyping and serotyping of Listeria monocytogenes. J. Clin. Microbiol. 39:2704-2707.

Nesbakken, T., G. Kapperud, and D. Caugant. 1996. Pathways of Listeria monocytogenes contamination in the meat processing industry. Int. J. Food Microbiol. 31:161-171.

Norton, D., M. McCamey, K. Gall, J. Scarlett, K. Boor, and M. Wiedmann. 2001a. Molecular studies on the ecology of Listeria monocytogenes in the smoked fish processing industry. Appl. Environ. Microbiol. 67:198-205.

Norton, D. M., J. M. Scarlett, K. Horton, D. Sue, J. Thimothe, K. J. Boor, and M. Wiedmann. 2001b. Characterization and pathogenic potential of Listeria monocytogenes isolates from the smoked fish industry. Appl. Environ. Microbiol. 67:646-653.

Piffaretti, J.-C., H. Kressebuch, M. Aeschenbacher, J. Bille, E. Bannerman, J. M. Musser, R. K. Seelander, and J. Rocourt. 1989. Genetic characterization of clones of the bacterium Listeria monocytogenes causing epidemic disease. Proc. Natl. Acad. Sci. USA 86:3818-3822.

Portnoy, D. A., T. Chakraborty, W. Goebel, and P. Cossart. 1992. Molecular determinants of Listeria monocytogenes pathogenesis. Infect. Immun. 60:1263-1267.

Pritchard, T. J., K. J. Flanders, and C. W. Donnelly. 1995. Comparison of the incidence of Listeria on equipment versus environmental sites within dairy processing plants. Int. J. Food Microbiol. 26:375-384.

Public Health Service/Food and Drug Administration. 1999. Grade "A" Pasteurized Milk Ordinance. U.S. Dept. of Health and Human Services, Public Health Service. Publication \#229. 292 pp. Washington, DC.

Rasmussen, O. F., T. Beck, J. E. Olsen, L. Dons, and L. Rossen. 1991. Listeria monocytogenes isolates can be classified into two major types according to the sequence of the listerioloysin gene. Infect. Immun. 59:3945-3951.

Rasmussen, O. F., P. Skouboe, L. Dons, L. Rossen, and J. E. Olsen. 1995. Listeria monocytogenes exists in at least three evolutionary lines: Evidence from flagellin, invasive associated protein and listeriolysin O genes. Microbiology 141:2053-2061.

Rorvik, L., D. Caugant, and M. Yndestad. 1995. Contamination pattern of Listeria monocytogenes and other Listeria spp. in a salmon 
slaughterhouse and smoked salmon processing plant. Int. J. Food Microbiol. 25:19-27.

Ryser, E. T. 1999a. Incidence and behaviour of Listeria monocytogenes in cheese and other fermented dairy products. Pages 411-503 in Listeria, Listeriosis, and Food Safety. E. T. Ryser and E. H. Marth, ed. Marcel Dekker Inc, New York.

Ryser, E. T. 1999b. Incidence and behaviour of Listeria monocytogenes in unfermented dairy products. Pages 359-409 in Listeria, Listeriosis, and Food Safety. E. T. Ryser and E. H. Marth, eds. Marcel Dekker, Inc., New York.

Sauders, B. D., K. G. Evans, K. Nightingale, and M. Wiedmann. 2002. Isolation and molecular subtyping of Listeria monocytogenes from pristine, urban, and agricultural sources. Abstract, International Conference on Emerging Infectious Diseases.

Schlech, W. F., P. M. Lavigne, R. A. Bortolussi, A. C. Allen, E. V. Haldane, A. J. Wort, A. W. Hightower, S. E. Johnson, S. H. King, E. S. Nicholls, and C. V. Broome. 1983. Epidemic listeriosis evidence for transmission by food. N. Engl. J. Med. 308:203-206.

Schwartz, B., D. Hexter, C. V. Broome, A. W. Hightower, R. B. Hirschhorn, J. D. Porter, P. S. Hayes, W. F. Bibbs, B. Lorber, and D. G. Faris. 1989. Investigation of an outbreak of listeriosis: new hypotheses for the etiology of epidemic Listeria monocytogenes infections. J. Infect. Dis. 159:680-685.

Seeliger, H. P. R. 1961. Listeriosis in animals. Pages 60-111 in Listeriosis. H. P. R. Seeliger, ed. Hafner Publishing Co., Inc., New York.

Stencel, C. 2000. Microbial diversity: Eyeing the big picture. ASM News 66:142-146.

Sutherland, P., and R. Porritt. 1996. Dissemination and ecology of Listeria monocytogenes in Australian dairy factory environments. Food Australia 48:172-178.
Tauxe, R. 1997. Emerging foodborne diseases: An evolving public health challenge. Emerg. Infect. Dis. 3:425-434.

Vines, A., M. W. Reeves, S. Hunter, and B. Swaminathan. 1992. Restriction fragment length polymorphism in four virulence-associated genes of Listeria monocytogenes. Res. Microbiol. 143:281-294.

Wesley, I. V. 1999. Listeriosis in animals. Pages 39-73 in Listeria, Listeriosis, and Food Safety. E. T. Ryser and E. H. Marth, ed. Marcel Dekker Inc., New York.

WHO. 1999. Food safety: an essential public health issue for the next millenium. World Health Organization, Geneva.

Wiedmann, M. 2002a. Molecular subtyping methods for Listeria monocytogenes. J. Assoc. Off. Anal. Chem. 85:524-531.

Wiedmann, M. 2002b. Subtyping technologies for bacterial foodborne pathogens. Nutr. Rev. 60:201-208.

Wiedmann, M., T. Arvik, J. L. Bruce, F. del Piero, M. C. Smith, J. Hurley, H. O. Mohammed, and C. A. Batt. 1997. An outbreak investigation of listeriosis in sheep in New York state. Am. J. Vet. Res. 58:733-737.

Wiedmann, M., J. L. Bruce, C. Keating, A. E. Johnson, P. L. McDonough, and C. A. Batt. 1997. Ribotypes and virulence gene polymorphisms suggest three distinct Listeria monocytogenes lineages with differences in pathogenic potential. Infect. Immun. 65:2707-2716

Wiedmann, M., J. L. Bruce, R. Knorr, M. Bodis, E. M. Cole, C. I. McDowell, P. L. McDonough, and C. A. Batt. 1996. Ribotype diversity of Listeria monocytogenes strains associated with outbreaks of listeriosis in ruminants. J. Clin. Microbiol. 34:1086-1090.

Wiedmann, M., and K. Evans. 2002. Infectious diseases of dairy animals: Listeriosis. Pages 777-778 in Encyclopedia of Dairy Science. H. Roginski, J. W. Fuquay, and P. F. Fox, ed. Academic Press, London. 